\section{Single-component quasicrystalline nanocrystal superlattices through flexible polygon tiling rule}

\author{
Yasutaka Nagaoka', Hua Zhu', Dennis Eggert ${ }^{2,3}$, Ou Chen ${ }^{1 *}$ \\ Quasicrystalline superlattices (QC-SLs) generated from single-component colloidal \\ building blocks have been predicted by computer simulations but are challenging to \\ reproduce experimentally. We discovered that 10 -fold QC-SLs could self-organize from \\ truncated tetrahedral quantum dots with anisotropic patchiness. Transmission electron \\ microscopy and tomography measurements allow structural reconstruction of the \\ QC-SL from the nanoscale packing to the atomic-scale orientation alignments. The unique \\ QC order leads to a tiling concept, the "flexible polygon tiling rule," that replicates the \\ experimental observations. The keys for the single-component QC-SL formation were \\ identified to be the anisotropic shape and patchiness of the building blocks and the \\ assembly microscopic environment. Our discovery may spur the creation of various \\ superstructures using anisotropic objects through an enthalpy-driven route.
}

A quasicrystalline $(\mathrm{QC})$ order possesses a striking rotational symmetricity, yet it has no transitional periodicity $(1,2)$. The first experimental discovery of a $\mathrm{QC}$ structure was made by Shechtman et al., who observed a selected-area electron diffraction pattern of Al-Mn alloys with a 10-fold rotational symmetry, which was strictly forbidden for periodic crystals (I). Soon after, scientists discovered 8-, 10-, and 12-fold two-dimensional (2D) polygonal QC structures and 3D icosahedral quasicrystals in a variety of intermetallic alloys (3). Subsequently, these findings led to studies that spread over a wide spectrum of research fields, including chemistry, materials science, mathematics, and even the arts (3-6).

Superstructures created by self-assembling objects at different length scales have attracted a great deal of scientific attention (7-11). Seminal discoveries of these fascinating structures have been presented using molecular and nanoscale building objects (12-17). However, examples of $\mathrm{QC}$ structures and their approximant orders created through bottom-up assemblies are still rare because of their metastability and high structural complexity. Wasio et al. reported a supramolecular assembly of a ferrocene derivative with a 10-fold QC order (7). Designed polymers have proved to be capable of self-organizing into 12-fold QC orders $(18,19)$. Talapin et al. pioneered the work of assembling inorganic colloidal nanocrystals (NCs) into 12-fold QC superlattices (SLs) using two different kinds of NCs with wellcontrolled size ratios (8). In the similar binary NC systems, Murray and co-workers proposed

\footnotetext{
${ }^{1}$ Department of Chemistry, Brown University, Providence, RI 02912, USA. ${ }^{2}$ Max Planck Institute for the Structure and Dynamics of Matter, Hamburg 22761, Germany. ${ }^{3}$ Heinrich Pette Institute-Leibniz Institute for Experimental Virology, Hamburg 20251, Germany.

*Corresponding author. Email: ouchen@brown.edu
}

new partial matching rules for generating $\mathrm{QC}$ SLs with structural consistency over a large area (10). We reported 2D QC-approximant SLs and $3 \mathrm{D}$ cluster-based supercrystals assembled from truncated tetrahedral quantum dots (TTQDs) (9). In addition to the experimental efforts, computer simulations have had a role in theorizing $\mathrm{QC}$ order formations (10, 20-22). Molecular dynamics computer simulations found a strong tendency of QC order formations from single-component colloids (20-22). Glotzer and colleagues showed that roughly spherical solids could be assembled into structures as complex as a 3D icosahedral quasicrystal $(20,21)$. Despite these advances, QC structures from single-component NC building blocks were not explored experimentally, leaving $\mathrm{NC}$ assemblies behind atomic crystals and computer simulations.

Here, we report the formation of large-area, 10-fold QC-SLs assembled from single-component TTQDs. Transmission electron microscopy (TEM) and tomography reconstruction allowed us to make direct observations of the $\mathrm{QC}$ structures, from the NC packing to the atomic orientations of the individual particles in real space. In conjunction with small-angle electron diffraction (SA-ED) and fast Fourier transform (FFT) analysis in reciprocal space, we found that the SL possessed a unique type of $\mathrm{QC}$ order. This discovery led us to propose a tiling concept Mechanistic studies demonstrated that the anisotropic surface tethers induced enthalpic patchiness of the TTQDs, which, combined with molecular microscopic environments at the assembly site, were responsible for the 10-fold QC-SL formation. Our discovery shows the possibility of creating superstructural materials, otherwise inaccessible through isotropic counterparts, from anisotropic building blocks even without the guidance of a unit cell. that we call the "flexible polygon tiling rule."
We synthesized wurtzite (WZ) TTQDs with a truncated tetrahedron shape, in which the three $\{10 \overline{1} 1\}_{\text {WZ }}$ major facets were coated with oleic acid and one $\{0002\}_{W Z}$ facet was passivated by octadecylphosphonic acid (ODPA) (Fig. 1A and figs. S1 and S2) (23). The inorganic height of the TTQD was $6.7 \pm 0.4 \mathrm{~nm}$ along the [0002 $]_{\mathrm{WZ}}$ crystal direction. We assembled the TTQDs by a liquid/air interface method (24), in which we slowly evaporated ( $\sim 6$ hours) a cyclohexane solution that contained TTQDs $(5.0 \mathrm{mg} / \mathrm{ml})$ on the surface of an ethylene glycol (EG) liquid subphase (Fig. 1B). TEM measurements of the resulting thin films indicated continuous SLs with lateral dimensions up to $\sim 50 \mu \mathrm{m}$ (Fig. 1B and figs. S3 and S4), but we did not identify translational periodicity (Fig. 1C). The SA-ED measurements we conducted were consistent with the real-space observations (Fig. 1C and figs. S3 to S5) as they displayed a diffraction spot pattern with a 10 -fold rotational symmetry, suggesting a QC order. Detailed analysis of the ED pattern indicated that the major ED signals were located at 10-basis vectors of $[\cos (2 \pi n / 10), \sin (2 \pi n / 10)]$ (Fig. 1C, pink, and fig. S5) with an expanded pentagon at a "golden ratio" of $(1+\sqrt{5}) / 2=1.62$ (fig. S5 and table S1). We observed two sets of weak satellite peaks at 10-basis (Fig. 1C, orange) and 20-basis (Fig. 1C, blue) vectors of $[\cos (2 \pi n / 10+2 \pi / 20), \sin (2 \pi n /$ $10+2 \pi / 20)]$ and $[\cos (2 \pi n / 20+2 \pi / 40), \sin (2 \pi n /$ $20+2 \pi / 40)$ ], respectively (fig. S5 and table $\mathrm{S} 1$ ). This 10 -fold symmetrical diffraction pattern with the presence of higher-order electron reflections of the SL confirmed the $\mathrm{QC}$ order with a long-range structural consistency (Fig. 1C). Furthermore, the same $\mathrm{QC}$ superstructure was generated when using larger TTQD building blocks with an inorganic NC height of $8.5 \pm 0.4 \mathrm{~nm}$ (figs. S6 and S7), proving the reliability of the formation of QC-SLs.

In-depth examinations showed that the $\mathrm{QC}$ SLs we observed were patterned with decagonderivative polygonal units (Fig. 1C). We used high-resolution TEM (HRTEM) to determine the atomic orientations of individual TTQDs in one polygonal unit. In addition to the 10 different atomic domains that we identified along the polygonal framework, there were two overlapping TTQD atomic orientations in the center (Fig. 1D, fig. S8, and table S2). The HRTEM results and particle orientation simulations allowed us to reconstruct the TTQD packing model in each polygonal unit (Fig. 1E and fig. S8). In the model, the polygonal framework was formed by ring tetrahelices with ramifications (9), and the center was stacked vertically by two TTQDs (figs. S9 and S10). We found that all the TTQDs in the model had the preferred facet-to-facet alignment (i.e., $\{0002\}_{\mathrm{WZ}}$-to- $\{0002\}_{\mathrm{WZ}}$ and $\{10 \overline{1} 1\}_{\mathrm{WZ}}$-to$\{10 \overline{1} 1\}_{\mathrm{WZ}}$ ) (Fig. 1E and figs. S9 and S10). These results suggested that the formation of singlecomponent 10-fold QC-SLs was induced by the anisotropic patchiness of the TTQDs (induced by the different surface molecular coatings and the intrinsic crystal dipole), where directional enthalpic forces were in play for the nucleation and growth of the QC-SLs (9). 
To better identify the TTQD packing in three dimensions, we used electron tomography measurements and computational 3D reconstructions to resolve the geometrical positions of individual TTQDs inside the SL (movies S1 and S2). The left panels of Fig. 2, A to F, show six representative horizontal slices of the measured QC-SL at different vertical positions. Although the 10-fold rotational symmetry was retained across all of the reconstructed slices, a unique transition of the particle packing pattern was captured from the top to the bottom of the SL (fig. S11 and movies S1 and S2). The magnified reconstruction images (highlighted areas in the left panels of Fig. 2, A to F) show that the SL area contained six interconnected decagon-derivative units (the centers of each polygon units labeled by magenta color, Fig. 2G). In contrast to the appearance of the center particles in all slices, the surrounding polygonal framework pattern alternated from top to bottom as follows: 5-TTQD-pentagon in the top (first) slice (Fig. 2A), 10-TTQD-decagon in the second slice (Fig. 2B), and 5-TTQD-pentagon with a clockwise rotation of $36^{\circ}$ with respect to the top pentagon (first slice) in the third slice (Fig. 2C). The same alternating pattern was repeated in the fourth to sixth slices (Fig. 2, D to F), indicating an identical second deck of the assembled structure along the vertical direction of the SLs (Fig. 2H). We further confirmed this double-decker structure by the vertical reconstruction (side view) of the QC-SLs (Fig. 2I and movie S3), which displayed a four-layer TTQD stacking (two layers in each deck). On the basis of the TEM and electron tomographic results, we constructed a 3D computer model of the six interconnected, decagon-derivative polygons to represent the unique architecture of the assembly (Fig. 2, G and H). Both the horizontal and vertical slices obtained from the computer model proved the model's correctness by matching the tomographic images perfectly (Fig. 2 and fig. S12).

The 10-fold $\mathrm{QC}$ order that we observed does not fall into any category of reported decagonal $\mathrm{QC}$ orders. Thus, to better understand the observed $\mathrm{QC}$ order, we introduced a "flexible polygon tiling rule" tiling method. Analogous to the "phason flip" mechanism (25), in which the whole system gains additional stability through local configuration changes at the interface (25), the flexible polygon tiling imparts local stability by having a flexible interface. In detail, the rule is constituted as follows: (i) Orientationally rigid regular polygons with an even number $(n)$ of edges shall be packed densely in a 2D space; (ii) when packing, two neighboring polygons may overlap with one or two edges, but no more than two edges; (iii) when no edge or one edge is overlapping, the two polygons remain intact; (iv) when overlapping with two edges, the overlapped edges shall become flexible and transform into one straight edge (denoted as a "flexible edge"); (v) each polygon must have at least one flexible edge and at most $n / 2$ flexible edges; and (vi) the generated tiling pattern has a $\mathrm{QC}$ order with $n$-fold rotational symmetry (Fig. 3 ,
A and B, and fig. S13). Note that because edge sharing while maintaining orientational rigidity is impossible for the polygons with an odd number of edges, this tiling rule is limited to the even-number-edge polygons. We verified this rule by using octagons, decagons, dodecagons, and tetradecagons as building units. We successfully obtained 8-, 10-, 12-, and 14-fold QC orders, respectively, as confirmed by the corresponding FFT patterns (figs. S14 to S17).

To apply this proposed $\mathrm{QC}$ tiling rule, we first extracted the center of each polygon in a 10-fold
QC-SL TEM image, and then quantified the nearest (Fig. 3C, yellow lines) and the second nearest (Fig. 3C, blue lines) inter-polygon center distances to be $16.6 \pm 0.4 \mathrm{~nm}$ and $19.5 \pm 0.5 \mathrm{~nm}$, respectively (Fig. 3D). This distance ratio was matched by the geometry-determined ratio of $(\cos 2 \pi / 10) /(\cos 2 \pi / 20)=0.8507$ for the decagonal center-to-center distances of one-edge (sharing one regular decagon edge) and twoedge (sharing one "flexible edge") overlapping scenarios (Fig. 3A and fig. S18). Consequently, the center of each polygon with $m$ "flexible edges"
A

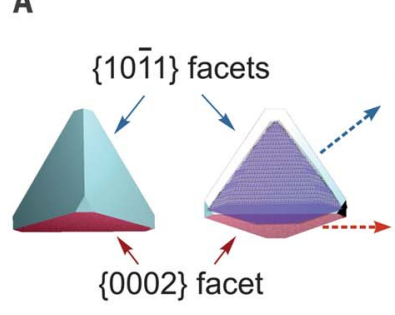

Oleic Acid

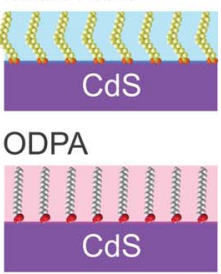

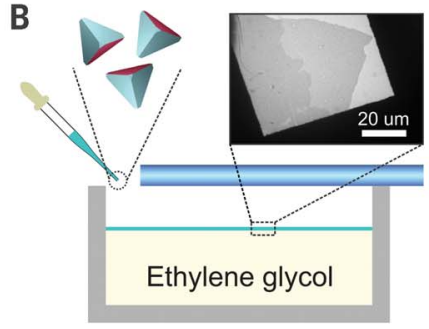
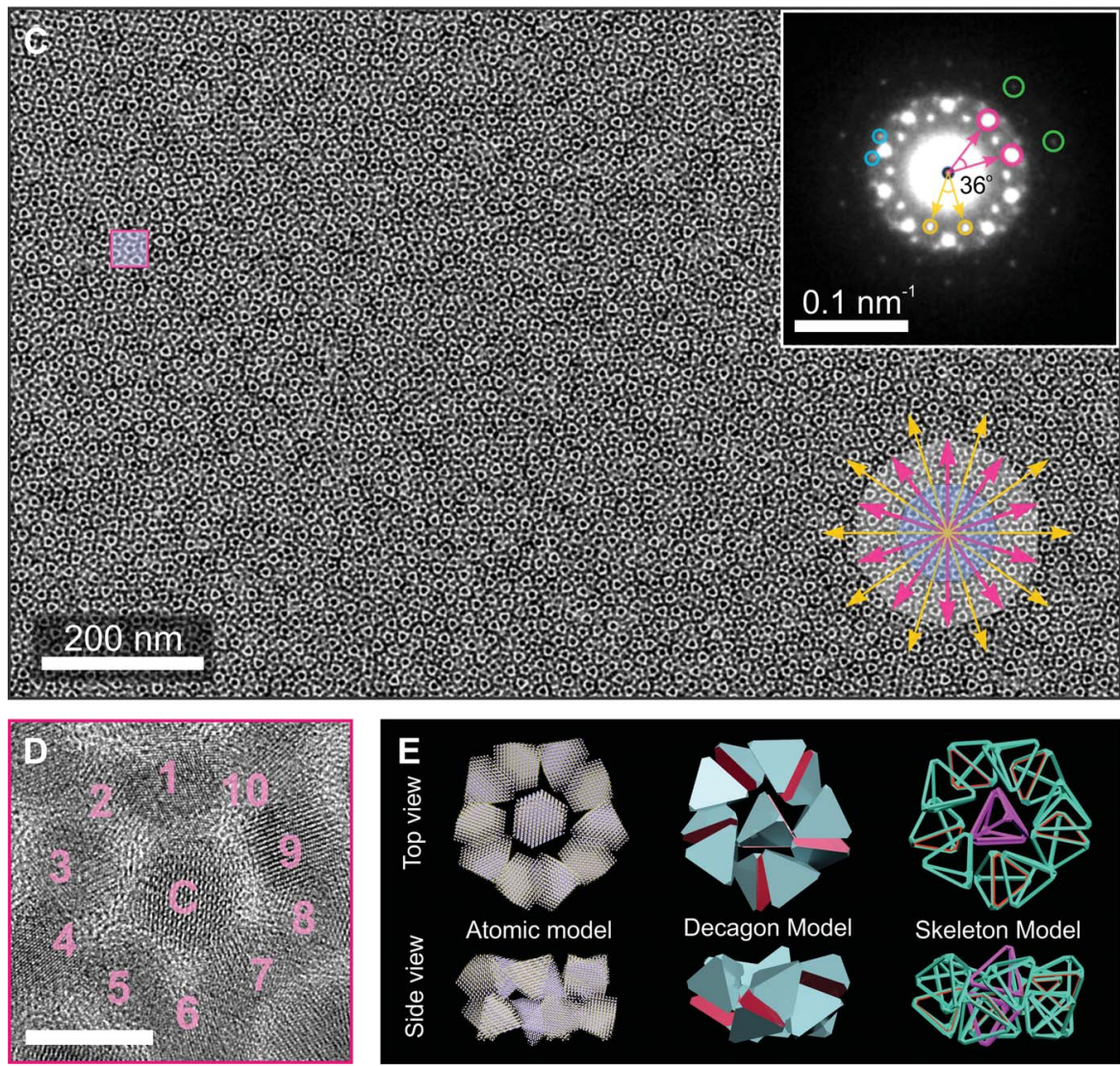

Fig. 1. 10-fold QC-SLs assembled from TTQDs. (A) Schematic illustration of an effective tetrahedral shape of a TTQD. The TTQD exhibits three major $\{10 \overline{1} 1\}$ facets coated with oleic acid (blue) and one $\{0002\}$ facet coated with ODPA (red). (B) Schematic illustration of the QC-SL formation at the liquid/air interface. (C) Representative TEM image of 10-fold QC-SLs and the corresponding SA-ED pattern (inset: pink, orange, and green cycles indicate 10-basis vectors; blue cycles indicate 20-basis vectors). (D) HRTEM image of a decagonderivative unit [square highlighted in $(\mathrm{C})$ ] with 10 atomic domains on the framework and 2 in the center. Scale bar, $10 \mathrm{~nm}$. (E) Computer-generated models (top and side views) of a decagon-derivative unit. 
Fig. 2. Tomography reconstruction of the QC-SL with a double-decker stacking. (A to F) Horizontal slices at six different vertical positions showing the NC arrangement within the first to sixth layers shown in $(H)$. Left: Snapshots of the tomographic movie. Center: Zoom-in images of the square-highlighted areas in left panels. Right: The corresponding horizontal slices from the computergenerated model shown in $(G)$ and $(H)$. (G and $\mathbf{H})$ A computer-generated model of a double-decker QC-SL with six interconnected decagon-derivative units shown from a top view (G) and a side view (H). The centers of each polygon unit are labeled in magenta. (I) A vertical reconstruction slice of a double-decker QC-SL showing a four-layer TTQD stacking in the vertical direction (from top to bottom: green, blue, red, and yellow). Scale bar, $30 \mathrm{~nm}$.
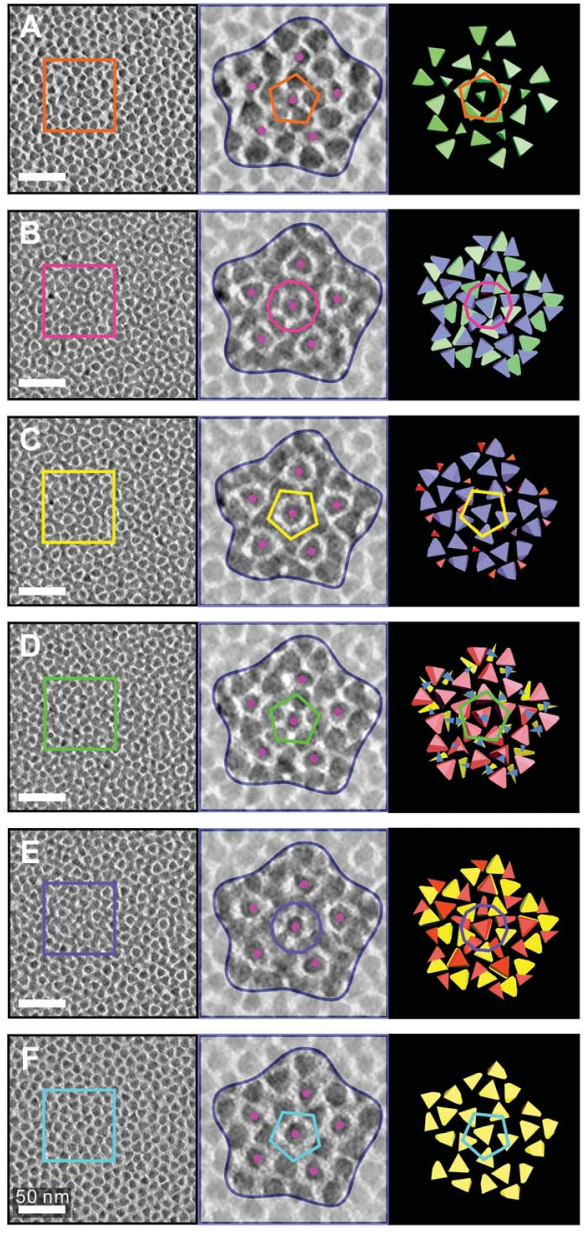
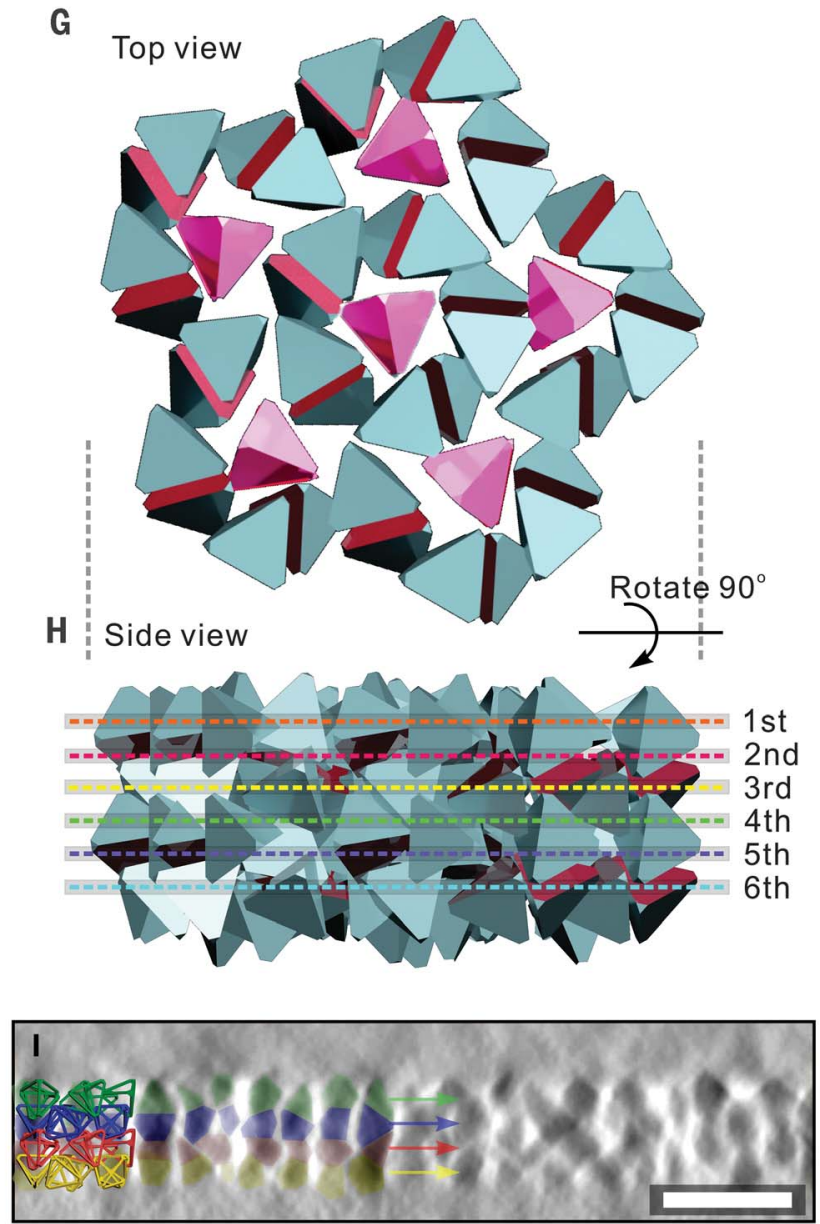

would be tiled by a $(10-m)$-edge polygon with a uniform orientation. Following this procedure, a 2D TEM image of the QC-SLs was tiled by "flexible" polygons with five to nine edges (Fig. $3 \mathrm{C}$ and fig. S19). Consistently, the FFT of the obtained tiling pattern showed a high degree of consistency with the measured SA-ED pattern and the FFT pattern of the corresponding TEM image (Fig. 3, E to G). In addition, we analyzed the angle distributions of the inter-polygon center directions. The angle distributions for both cases (one-edge and two-edge overlapping cases) showed a 10 -fold rotational symmetry (Fig. $3 \mathrm{H}$ ), yet there was an angle offset of $18.0^{\circ} \pm 1.8^{\circ}$ between the two cases (Fig. $3 \mathrm{H}$ and fig. S20). This angle analysis statistic was consistent with an overall 10-fold symmetry of the obtained TTQD assembled QC-SLS, which further validated our flexible polygon tiling rule.

Studies using silica microbeads have shown that patchy colloids, as a result of their unique energetic characteristics (vibrational and rotational entropies), can self-organize into unconventional structures more favorably than conventional lattices with equivalent potential energy upon crowding $(26,27)$. In our case, the shape of the particles and their associated anisotropic surface patchiness (i.e., ODPA on $\{0002\}_{\mathrm{WZ}}$ and oleic acid on $\{10 \overline{1} 1\}_{\mathrm{WZ}}$ ) proved to be mandatory in order to form the observed QC-SLs (9). When assembling the isotropic analogs (oleic acidcoated spherical QDs) with the same singleparticle volume, a regular face-centered cubic SL was obtained (fig. S21). In addition, the selection of the liquid subphase was found to be vital $(24,28)$. In contrast to the 10 -fold QC-SLs, a stack of monolayer hexagonal SLs was generated when EG was replaced with diethylene glycol (DEG) (figs. S22 to S24 and movies S4 and S5). This likely was due to the differences in their molecular structures; only the hydroxyl group is present in EG, whereas DEG contains an additional ether group, which provided a higher aliphatic Hansch hydrophobicity $(\pi=$ -0.71 for $-\mathrm{COCH}_{3}$ versus -1.16 for $\left.-\mathrm{OH}\right)(29,30)$. The smaller $\pi$ value for EG resulted in a lower interfacial surface energy (i.e., lower intermolecular affinity) between the solvent (cyclohexane) and the substrate (EG) (31), leading to a higher receding angle at the evaporation microsite (i.e., the SL formation site; see supplementary text) $(32,33)$. Consequently, the TTQDs could interact with each other freely in space, forming the 10-fold QC-SLs in a process that largely was driven by the directional enthalpic patchiness (Fig. 4, i) $(9,27)$. However, changing the liquid subphase to
DEG resulted in a larger $\pi$ value and thus a lower receding angle at the evaporation site, which limited the assembly space in the vertical dimension, thereby diminishing the interparticle enthalpic interactions. In addition, the same larger $\pi$ value translated to a stronger attractive Derjaguin-Landau-Verwey-Overbeek force, thereby inducing a higher affinity between the ligand molecules on the surfaces of the TTQDs (i.e., oleic acid or ODPA) and the DEG liquid subphase, further minimizing the inter-TTQD interactions. Together, the formation of a hexagonal TTQD monolayer, dominantly driven by entropy, was favored (Fig. 4, ii). Consequently, upon further stacking, multilayer hexagonal SL generation was obtained (Fig. 4, ii, and fig. S25) (32, 33). Likewise, 10-fold QC-SLs were formed on the surface of the glycerol, whereas a stack of monolayer hexagonal SLs was generated on top of the tri- and tetra-EG, thereby validating the reliability of the formation mechanism (fig. S25).

Our findings show that high-complexity superstructures can be obtained from single-component NC building blocks when anisotropic kinetic factors are fueled. We anticipate that the newly discovered QC-SLs and the proposed "flexible polygon" aperiodic tiling rule will spur other interesting 
Fig. 3. QC order generated through a flexible polygon tiling rule. (A) Schematic illustration of the flexible edge transformation process for two-edge overlapping between two regular decagons. (B) Schematic demonstration of a flexible decagon transforming into possible orientational rigid polygons with nine to five edges.

(C) A TEM image showing the flexible polygon tiling steps. Larger orange spheres at top left indicate the centers of each polygon; the yellow and blue lines connect the two polygon centers with the nearest and second nearest inter-polygon center distance, respectively; the right side of the image is tiled by color-coded flexible polygons [same color coding as in (B)]. (D) Histograms of the length distributions of the nearest (yellow lines) and second nearest (blue lines) inter-polygon center distances. (E) FFT pattern of an artificial QC order generated from flexible polygon tiling as shown in (C). (F) SA-ED pattern obtained from the TTQD QC-SLs. (G) FFT pattern of a 10-fold QC-SL TEM image. (H) Angle distributions of the nearest (yellow) and second nearest (blue) inter-polygon center directions.

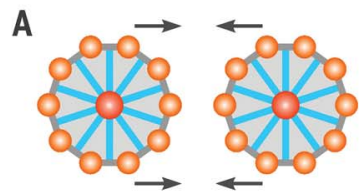

Neighboring decagons

B Decagon

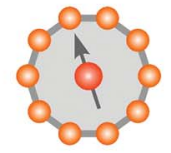

10-edge

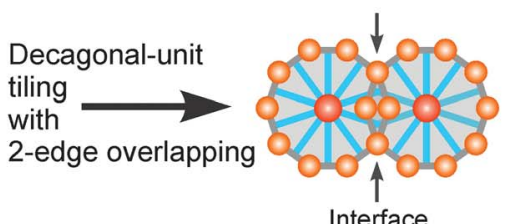

Interface Nonagon

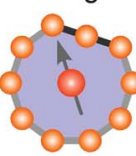

9-edge

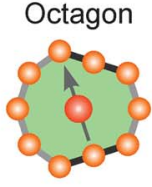

8-edge
C

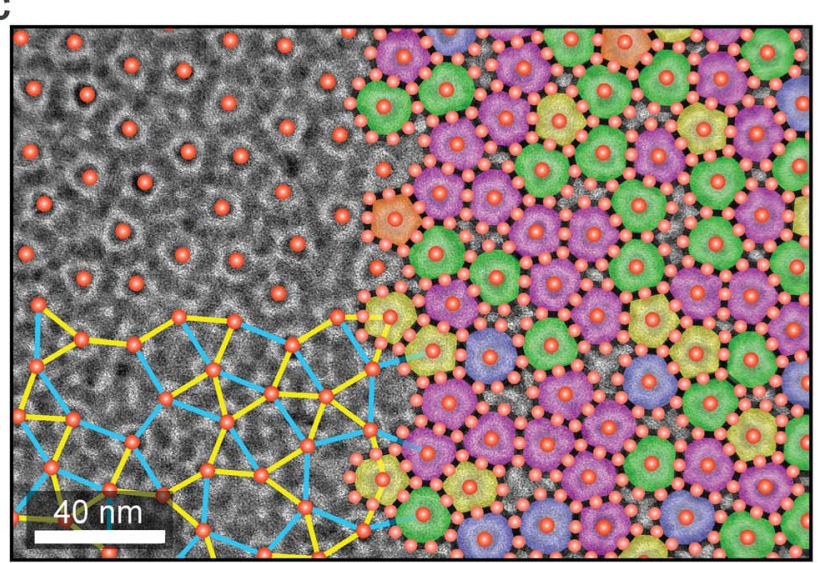

D

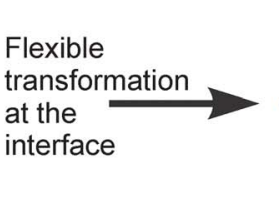

Heptagon

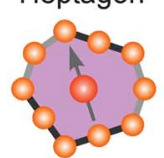

7-edge

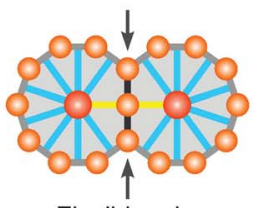

Flexible edge

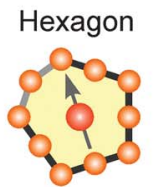

6-edge

Pentagon

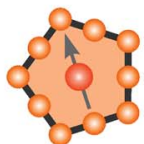

5-edge
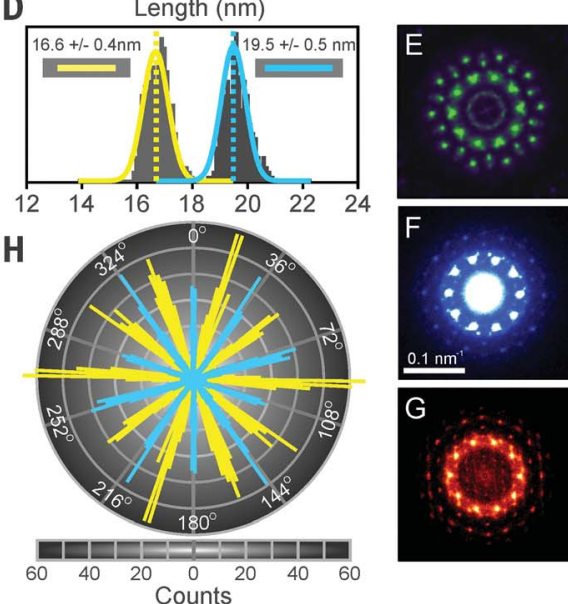

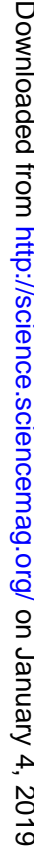
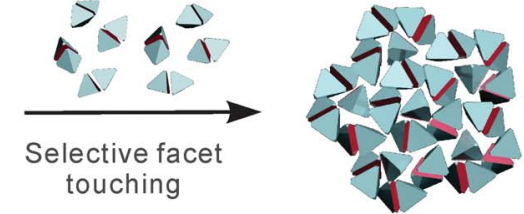

10-fold QCSL

SL formation evaporation site
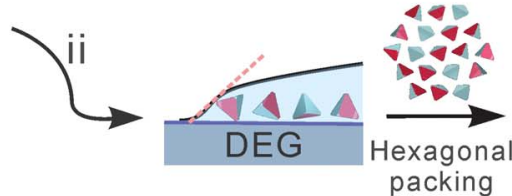

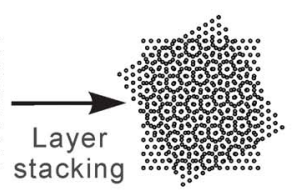

Hexagonal SL stacking

Fig. 4. Schematic representation of the TTQD assembly pathways. (i) 10-fold QC-SL formation on top of EG; (ii) hexagonal SL stacking formation on top of DEG.

superstructural assemblies from a spectrum of anisotropic building blocks of various length scales.

\section{REFERENCES AND NOTES}

1. D. Shechtman, I. Blech, D. Gratias, J. W. Cahn, Phys. Rev. Lett. 53, 1951-1953 (1984).
2. D. L. D. Caspar, E. Fontano, Proc. Natl. Acad. Sci. U.S.A. 93 , 14271-14278 (1996)

3. A. Yamamoto, Acta Crystallogr. A 52, 509-560 (1996).

4. W. N. Man, M. Megens, P. J. Steinhardt, P. M. Chaikin, Nature 436, 993-996 (2005)

5. W. Steurer, Chem. Soc. Rev. 41, 6719-6729 (2012)

6. P. J. Lu, P. J. Steinhardt, Science 315, 1106-1110 (2007).

7. N. A. Wasio et al., Nature 507, 86-89 (2014).
8. D. V. Talapin et al., Nature 461,964-967 (2009).

9. Y. Nagaoka et al., Nature 561, 378-382 (2018).

10. X. Ye et al., Nat. Mater. 16, 214-219 (2017)

11. Z. J. Yang, J. J. Wei, P. Bonville, M. P. Pileni, J. Am. Chem. Soc. 137, 4487-4493 (2015)

12. H. X. Lin et al., Science 355, 931-935 (2017)

13. M. P. Boneschanscher et al., Science 344, 1377-1380 (2014)

14. M. Yang et al., Nat. Chem. 9, 287-294 (2017).

15. Z. J. Yang, J. J. Wei, Y. I. Sobolev, B. A. Grzybowski, Nature 553, 313-318 (2018)

16. K. Miszta et al., Nat. Mater. 10, 872-876 (2011)

17. R. M. Choueiri et al., Nature 538, 79-83 (2016).

18. X. B. Zeng et al., Nature 428, 157-160 (2004).

19. K. Hayashida, T. Dotera, A. Takano, Y. Matsushita, Phys. Rev. Lett. 98, 195502 (2007).

20. A. Haji-Akbari et al., Nature 462, 773-777 (2009).

21. M. Engel, P. F. Damasceno, C. L. Phillips, S. C. Glotzer, Nat. Mater. 14, 109-116 (2015).

22. T. Dotera, T. Oshiro, P. Ziherl, Nature 506, 208-211 (2014).

23. R. Tan et al., Chem. Mater. 29, 4097-4108 (2017).

24. A. Dong, J. Chen, P. M. Vora, J. M. Kikkawa, C. B. Murray, Nature 466, 474-477 (2010).

25. M. Engel, H.-R. Trebin, Phys. Rev. Lett. 98, 225505 (2007).

26. Q. Chen, S. C. Bae, S. Granick, Nature 469, 381-384 (2011)

27. V. N. Manoharan, Science 349, 1253751 (2015).

28. B. T. Diroll, N. J. Greybush, C. R. Kagan, C. B. Murray, Chem. Mater. 27, 2998-3008 (2015).

29. D. Guo, C. Li, Y. Wang, Y. Li, Y. Song, Angew. Chem. Int. Ed. 56 15348-15352 (2017).

30. A. Leo, C. Hansch, D. Elkins, Chem. Rev. 71, 525-616 (1971) 
31. F. M. Menger, M. E. Chlebowski, Langmuir 21, 2689-2695 (2005).

32. S. Šikalo, H. D. Wilhelm, I. V. Roisman, S. Jakirlic, C. Tropea, Phys. Fluids 17, 062103 (2005).

33. R. Bhardwaj, X. H. Fang, P. Somasundaran, D. Attinger, Langmuir 26, 7833-7842 (2010)

\section{ACKNOWLEDGMENTS}

O.C. thanks S. Zhao for valuable discussion. The TEM measurements were performed at the Electron Microscopy Facility in the Institute for Molecular and Nanoscale Innovation
(IMNI) at Brown University. Funding: Supported by the Brown University Startup Fund and the IMNI Seed Fund (O.C.). Author contributions: Y.N. and O.C. conceived and designed the experiments; Y.N. and H.Z. conducted the synthesis and material characterizations; Y.N. performed the data analysis; D.E. performed electron tomography measurement and computer reconstruction; O.C. supervised the entire project; and Y.N. and O.C. wrote the manuscript. All authors discussed the results and commented on the manuscript. Competing interests: The authors declare no competing financial interest. Data and materials availability: All data are available in the main text or the supplementary materials.

\section{SUPPLEMENTARY MATERIALS}

www.sciencemag.org/content/362/6421/1396/suppl/DC1

Materials and Methods

Supplementary Text

Figs. S1 to S25

Tables S1 and S2

Movies S1 to S5

References (34-41)

15 August 2018; accepted 24 October 2018 10.1126/science.aav0790 


\section{Science}

\section{Single-component quasicrystalline nanocrystal superlattices through flexible polygon tiling rule}

Yasutaka Nagaoka, Hua Zhu, Dennis Eggert and Ou Chen

Science 362 (6421), 1396-1400.

DOI: $10.1126 /$ science.aav0790

\section{Quantum dots line up as a quasicrystal}

Quasicrystals have rotational symmetry but no long-range order. Although several materials have quasicrystalline order, examples of quasicrystalline superlattices formed from different types of particles are rare. Nagaoka et al. discovered a type of 10 -fold quasicrystalline superlattice formed from truncated tetrahedral quantum dots (see the Perspective by Wu and Sun). The order is driven by a "flexible polygon tiling rule," which explains the unique arrangement of the quantum dots.

Science, this issue p. 1396; see also p. 1354

ARTICLE TOOLS

SUPPLEMENTARY MATERIALS

RELATED

CONTENT

REFERENCES

PERMISSIONS http://science.sciencemag.org/content/362/6421/1396

http://science.sciencemag.org/content/suppl/2018/12/19/362.6421.1396.DC1

http://science.sciencemag.org/content/sci/362/6421/1354.full

This article cites 40 articles, 5 of which you can access for free http://science.sciencemag.org/content/362/6421/1396\#BIBL

http://www.sciencemag.org/help/reprints-and-permissions

Use of this article is subject to the Terms of Service

Science (print ISSN 0036-8075; online ISSN 1095-9203) is published by the American Association for the Advancement of Science, 1200 New York Avenue NW, Washington, DC 20005. 2017 () The Authors, some rights reserved; exclusive licensee American Association for the Advancement of Science. No claim to original U.S. Government Works. The title Science is a registered trademark of AAAS. 